\title{
Atlas of the Postnatal Rat Brain in Stereotaxic Coordinates
}

\author{
Roustem Khazipov ${ }^{1,2,3 *}$, Dilyara Zaynutdinova ${ }^{1,2}$, Elena Ogievetsky ${ }^{1,2}$, Guzel Valeeva ${ }^{3}$, \\ Olga Mitrukhina ${ }^{1,2,3}$, Jean-Bernard Manent ${ }^{1,2}$ and Alfonso Represa ${ }^{1,2}$ \\ ${ }^{1}$ Institut de Neurobiologie de la Méditerranée, Institut National de la Santé et de la Recherche Médicale U-901, Marseille, \\ France, ${ }^{2}$ Aix-Marseille University, UMR 901, Marseille, France, ${ }^{3}$ Laboratory of Neurobiology, Kazan Federal University, Kazan, \\ Russia
}

Keywords: rat, neonate, brain, atlas, coordinates, bregma, lamda

\section{OPEN ACCESS}

Edited by:

Shawn Mikula

Max Planck Institute for Neurobiology,

Germany

Reviewed by:

Heiko J. Luhmann,

Institut für Physiologie und

Pathophysiologie, Germany

Zoltan Molnar,

University of Oxford, UK

Nuno Maçarico da Costa

Allen Institute for Brain Science, USA

*Correspondence:

Roustem Khazipov

roustem.khazipov@inserm.fr

Received: 19 October 2015 Accepted: 30 November 2015 Published: 23 December 2015

Citation:

Khazipov R, Zaynutdinova $D$,

Ogievetsky $E$, Valeeva $G$,

Mitrukhina O, Manent J-B and

Represa A (2015) Atlas of the

Postnatal Rat Brain in Stereotaxic

Coordinates. Front. Neuroanat. 9:161.

doi: 10.3389/fnana.2015.00161
The series of atlases of the developing rat brain in stereotaxic coordinates that we propose here ${ }^{1,2}$ has been conceived as a tool for the exploration of the rat brain in vivo during the postnatal period, offering a choice of bregma and lambda as the reference points. We suggest to use this atlas, which provides stereotaxic coordinates, in combination with other histological atlases, where brain structures and boundaries between them were assessed using histochemistry or quantative magnetic resonance techniques, but where stereotaxic coordinates are not available (Ramachandra and Subramanian, 2011; Calabrese et al., 2013; Ashwell and Paxinos, 2015) or provided (at P10, P21, and P39) but not within the bregma-lambda metrics (Sherwood and Timiras, 1970).

The neonatal rat is a powerful model for the study of the early stages of central nervous system development. Comparative developmental studies suggest that the first two postnatal weeks in the rat roughly correspond to the second half of gestation and the early postnatal period in humans, with the electrical brain activity patterns expressed during this period and their developmental trajectories matching the patterns observed in premature human neonates (Clancy et al., 2001, 2007; Khazipov and Luhmann, 2006; Colonnese et al., 2010; Workman et al., 2013) (see also http:// www.translatingtime.net/). Recent advances in the understanding of the developing brain functions largely came from the use of techniques to record brain activity from neonatal and adolescent rats in vivo, notably in head restrained animals (Leinekugel et al., 2002; Khazipov et al., 2004; Minlebaev et al., 2011; Tiriac et al., 2012; Yang et al., 2013). However, the rat brain grows rapidly and nonproportionally during the postnatal period and brain structures change their position in relation to the conventional skull marks such as bregma and lambda, making difficult to perform targeted recordings, stimulations, lesions, or local drug injections in precise anatomical locations. Hence the importance of a developmental atlas of the rat brain in stereotaxic coordinates.

Several examples of histology atlases series of the developing rat brain have been published (Table 1). The Atlas by Sherwood and Timiras "A Stereotaxic Atlas of the Developing Rat Brain" atlas includes three postnatal time points: postnatal days P10, P21, and P39 (Sherwood and Timiras, 1970). Ashwell and Paxinos' "Atlas of the Developing Rat Nervous System" provides photographs and accompanying diagrams of coronal and sagittal sections of rats aged E12, E13, E14, E16, E17, E19, and P0 (Ashwell and Paxinos, 2015). "Atlas of the Neonatal Rat Brain" by Ramachandra and Subramanian describes rat brains at P1, P7, and P14 (Ramachandra and Subramanian, 2011). More recently, Calabrese, Badea, Watson, and Johnson published their "Quantitative magnetic resonance histology atlas of postnatal rat brain development with regional estimates of growth and variability" based on the study of P0, P2, P4, P8, P12, P18, P24, P40, and P80 rats (Calabrese et al., 2013). Among these atlases, only the atlas by Sherwood and Timiras (1970) provides stereotaxic coordinates and

The Atlas of the Developing rat brain in stereotaxic coordinates has been registered at APP as a data base under a reference \# IDDN.FR.001.180010.000.D.P.2013.000.31230

${ }^{1}$ www.ial-developmental-neurobiology.com/en/publications/collection-of-atlases-of-the-rat-brain-in-stereotaxic-coordinates; ${ }^{2} \mathrm{http}: / /$ www.inmed.fr/en/en-atlas-stereotaxique-du-cerveau-de-rat-au-cours-du-developpement-postnatal. 


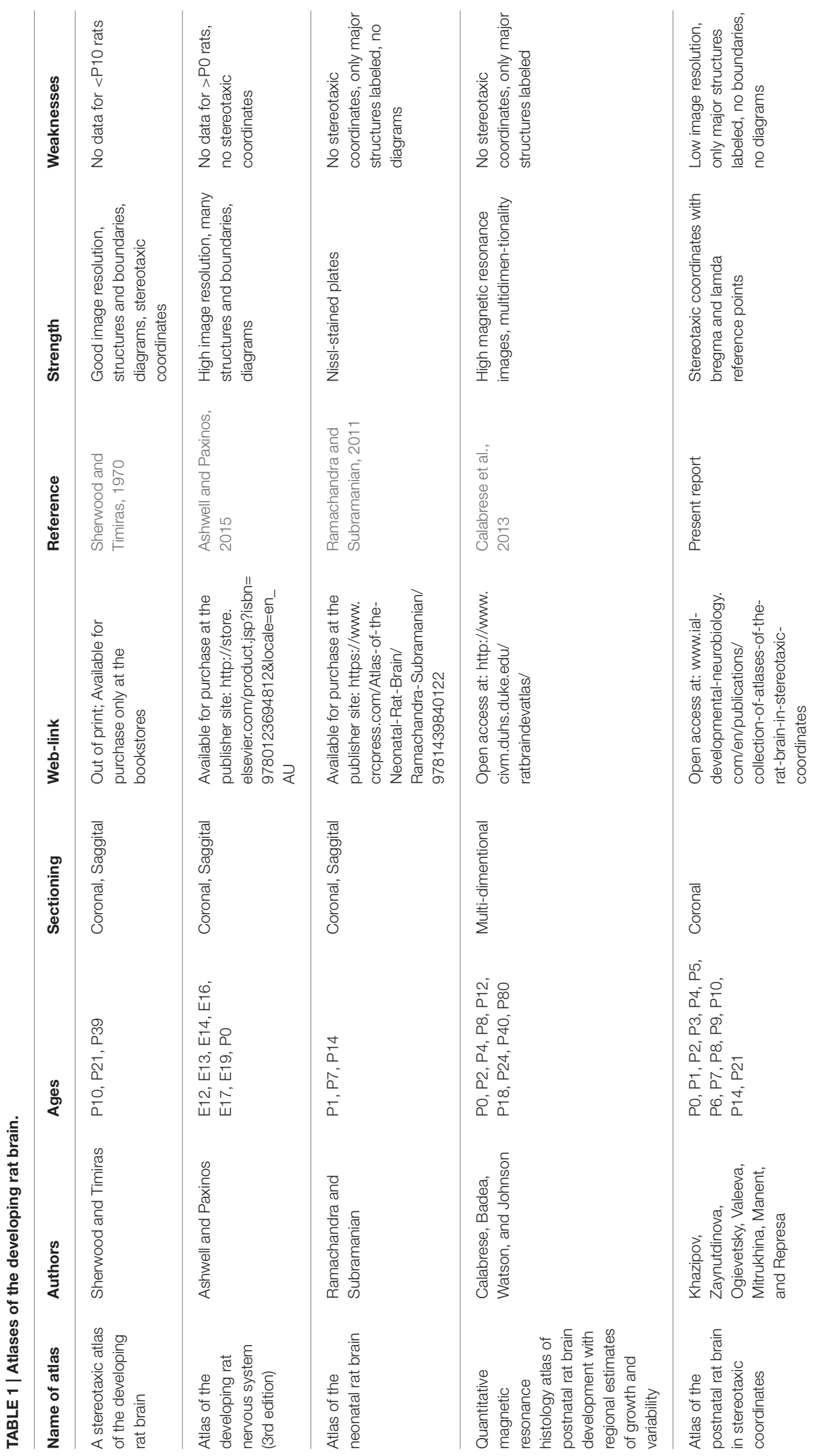


yet for only three postnatal days P10, 21, and P39. Stereotaxic atlases for the early postnatal period are not available, however.

The series of atlases of the developing rat brain in stereotaxic coordinates that we propose here has been conceived as a tool for the exploration of the rat brain in vivo during the postnatal period, offering a choice of bregma and lambda as the reference points. Each atlas contains a series of microphotographs of wet, non-stained 200 micron coronal brain sections in oblique light, obtained from postnatal days P0, 1, 2, 3, 4, 5, 6, 7, 8, 9, 10, 14, and 21 rats, with an indication of the main brain structures. Wet non-stained sections were used to avoid deformation, shrinkage and lessening associated with staining and mounting procedures, particularly when manipulating immature tissue. Because we did not stain sections, the images do not provide cellular resolution and not all brain structures and boundaries between them can be easily identified. Therefore, only certain brain structures are labeled without boundaries. Used in combination with the existing high image resolution atlases of the developing rat brain (Sherwood and Timiras, 1970; Ramachandra and Subramanian, 2011; Calabrese et al., 2013; Ashwell and Paxinos, 2015), this atlas may be useful for targeted recordings, stimulations, and drug injections in different brain structures at various postnatal ages. This atlas has been successfully used in several previous studies (Minlebaev et al., 2011; Petit et al., 2014; Tyzio et al., 2014).

\section{STEREOTAXIC SURGERY}

All animal-use protocols followed the guidelines of the French National Institute of Health and Medical Research (INSERM, protocol N007.08.01). Wistar rats of both sexes from postnatal days $[\mathrm{P}] 0,1,2,3,4,5,6,7,8,9,10,14$, and 21 were used. $\mathrm{P} 0$ was the day of birth. Three to five animals per age were used in this study and one animal per age with the best histology quality was selected for the atlas. The surgery was performed under urethane anesthesia $(1.5 \mathrm{~g} / \mathrm{kg})$. The skull of the animal was cleaned of skin and periosteum. A hemostabe was used to stop bleeding. Two metal bars were fixed to the nasal and occipital bones of the rat's head with dental cement (Grip Cement). During tracing, the head was fixed to the frame of the Kopf stereotaxic apparatus with bars attached similarly to the head fixation used during recordings from neonatal rat pups (Leinekugel et al., 2002; Khazipov et al., 2004; Minlebaev et al., 2011). This approach is different from the conventional method of head fixation with ear and tooth bars used by Sherwood and Timiras (1970), which cannot be used in neonatal animals $<\mathrm{P} 10$ because the skull is cartilaginous. Bregma and lambda were adjusted to the same height and laterally aligned using a needle attached to a stereotaxic manipulator to control their vertical and horizontal coordinates. Thus, the horizontal plane of the atlas extends from bregma to lambda. Because of the use of an "artificial skull" for the head fixation, and unlike in other stereotaxic atlases, we do not provide a vertical zero plane.

Holes were drilled in the skull $1-3 \mathrm{~mm}$ lateral from the bregma and lambda, through which a needle colored by dipping in an ethanol solution of DiI (1, $1^{\prime}$-Dioctadecyl-3,3,3',3'tetramethylindocarbocyanine perchlorate, Sigma-Aldrich, St. Louis, USA) was vertically inserted at a depth of 3-5 mm. An additional insertion of the DiI colored needle through the whole brain in a coronal plane was made $3-5 \mathrm{~mm}$ lateral from the midline between bregma and lambda. This line was used for the positioning of the brain during coronal slicing.

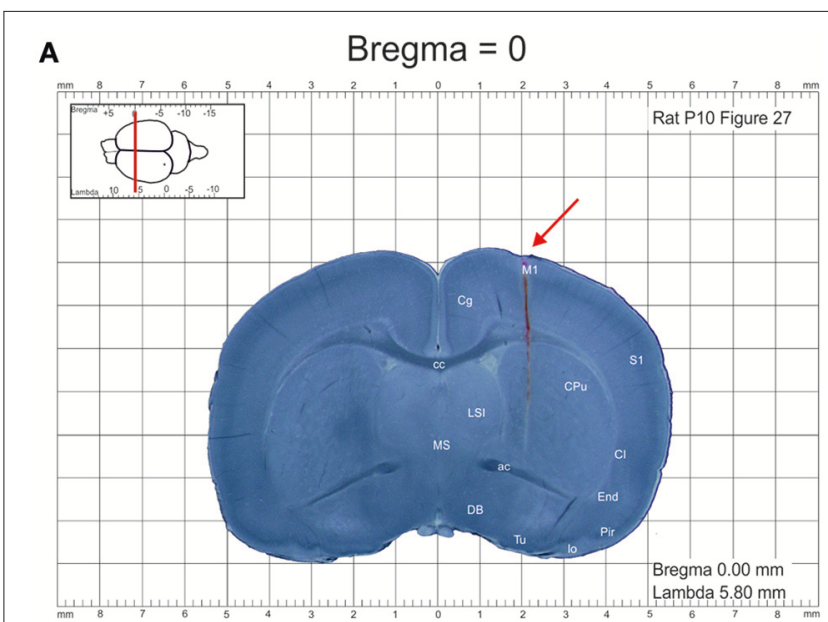

B
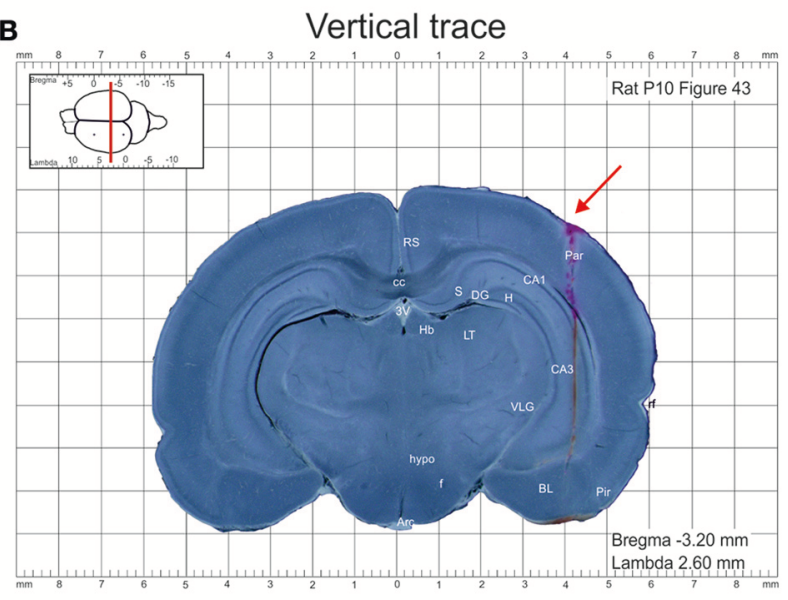

C
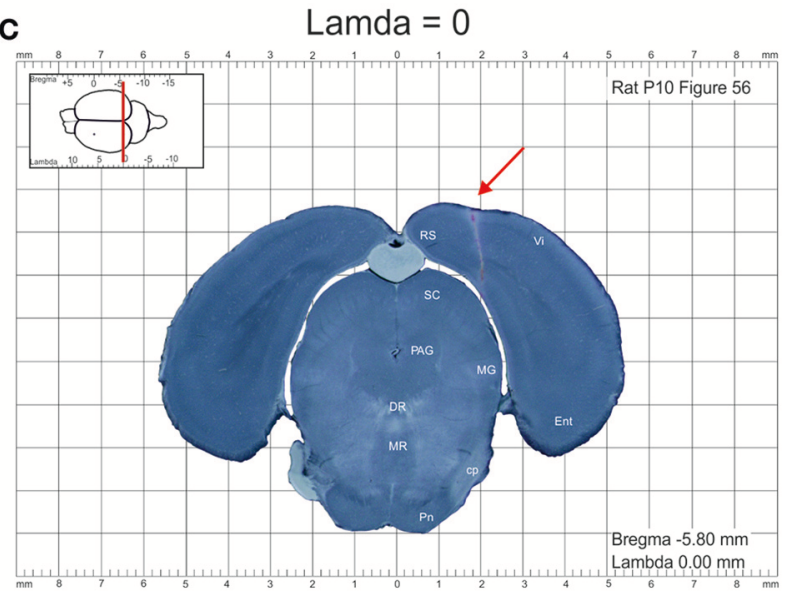

FIGURE 1 | Example images of the coronal sections from the postnatal day $\mathrm{P} 10$ rat brain from the atlas of the developing rat brain in stereotaxic coordinates. (A-C) Coronal sections at anterioposterior zero distances from the bregma (A) and lambda (C), and a section illustrating the trace through the whole brain (B). Dil-labeled penetrations are indicated by red arrows. 


\section{HISTOLOGY}

After tracing, rats were intracardially perfused with a solution of 4\% paraformaldehyde ( $\mathrm{pH} 7.2-7.4$; Antigenfix, Diapath, Italy) and $1-3 \%$ glutaraldehyde (Sigma-Aldrich, St. Louis, USA), the brain was removed and left in fixative for 1-6 days depending on the age of the animal. Then brains were rinsed in PBS (prepared from PBS tablets, Gibco, Paisley, UK; pH 7.45, osmolarity 305 mosm) photographed in DiI fluorescent light from above and placed in a block of agar. To ensure that coronal sections were made strictly perpendicular to the horizontal plane, blocks were cut at the bottom parallel to the lateral DiI-labeled trace going through the whole brain. Then the blocks were glued to the metal plate of the vibroslicer (Leica VT1000S) and 200 micron thick coronal sections were cut from the olfactory bulb to the spinal cord. Sections were then placed in a 12 wells plastic dish and kept in PBS. Microphotographs of wet sections were made using OLYMPUS SZX16 microscope equipped with a DP71camera, at 0.7-1.6 zoom in oblique light. During photography, the excess of PBS was removed. No coloration of brain sections was used in this study. Microphotographs were acquired at $150 \mathrm{dpi}$ in .psd format using cellSens Standard 1.6 software.

\section{IMAGE PROCESSING}

Images were further processed using Photoshop CS5.1 software and converted to 150 dpi images in .png format. DiI labeled tracks of the penetrations at bregma and lambda levels were used to calculate the anterioposterior distance of each section from the bregma and lambda, respectively (Figures 1A,C). The entire appearance of the lateral across-brain track in not more than two sections was used to ensure that the sections were cut parpendicular to the horizontal plane (Figure 1B). Otherwise, the brains were discarded from analysis. Images were further assembled into the atlas using InDesigner CS5.5 with an indication of the anterioposterior distance of the section from bregma and lamda and converted to the final.pdf format at 300 dpi resolution.

\section{REFERENCES}

Ashwell, K. W. S., and Paxinos, G. (2015). Atlas of the Developing Rat Nervous System, 3rd Edn. San Diego, CA: Elsevier Academic Press.

Calabrese, E., Badea, A., Watson, C., and Johnson, G. A. (2013). A quantitative magnetic resonance histology atlas of postnatal rat brain development with regional estimates of growth and variability. Neuroimage 71, 196-206. doi: 10.1016/j.neuroimage.2013.01.017

Clancy, B., Darlington, R. B., and Finlay, B. L. (2001). Translating developmental time across mammalian species. Neuroscience 105, 7-17. doi: 10.1016/S03064522(01)00171-3

Clancy, B., Finlay, B. L., Darlington, R. B., and Anand, K. J. (2007). Extrapolating brain development from experimental species to humans. Neurotoxicology 28, 931-937. doi: 10.1016/j.neuro.2007.01.014

Colonnese, M. T., Kaminska, A., Minlebaev, M., Milh, M., Bloem, B., Lescure, S., et al. (2010). A conserved switch in sensory processing prepares developing neocortex for vision. Neuron 67, 480-498. doi: 10.1016/j.neuron.2010. 07.015

\section{DATA BASE DESCRIPTION}

The collection of atlases prepared from postnatal days P0, 1, $2,3,4,5,6,7,8,9,10,14$, and 21 rats is deposited at www.ialdevelopmental-neurobiology.com/en/publications/collection-ofatlases-of-the-rat-brain-in-stereotaxic-coordinates and http:// www.inmed.fr/en/en-atlas-stereotaxique-du-cerveau-de-rat-aucours-du-developpement-postnatal. Each file contains a series of obtained in oblique light microphotographs of 200 micron wet, non-colored coronal brain sections of a rat at the age indicated in the file name and a list of abbreviations of the main brain structures. For identifying brain structures, sections were systematically compared with images from the developing rat brain atlases (Sherwood and Timiras, 1970; Paxinos et al., 1991; Ramachandra and Subramanian, 2011), adult rat brain atlas (Paxinos and Watson, 2007), and our own stained sections from the age-matching animals used in other studies. Only the brain areas that we could accurately identify were quoted in the study. Atlases are presented in pdf format at $300 \mathrm{dpi}$ resolution. To move along the anterioposterior sagittal plane one can use conventional Adobe view instruments or chose a position by clicking the brain image located at the left top panel.

\section{AUTHOR CONTRIBUTIONS}

RK conceived the project. RK, DZ, GV, and OM performed tracing and prepared brain sections. $\mathrm{DZ}$ made photographs of the brain sections. JM and AR marked brain regions. EO processed images and assembled the atlases. RK wrote the paper.

\section{ACKNOWLEDGMENTS}

This work was supported by INSERM (LIA to RK), FRM (DEQ20110421301 to RK), Ministry of Education and Science, the Government of the Russian Federation (11.G34.31.0075 to $\mathrm{RK})$, and the program of competitive growth of Kazan University (RK, OM, GV).

Khazipov, R., and Luhmann, H. J. (2006). Early patterns of electrical activity in the developing cerebral cortex of humans and rodents. Trends Neurosci. 29, 414-418. doi: 10.1016/j.tins.2006.05.007

Khazipov, R., Sirota, A., Leinekugel, X., Holmes, G. L., Ben Ari, Y., and Buzsaki, G. (2004). Early motor activity drives spindle bursts in the developing somatosensory cortex. Nature 432, 758-761. doi: 10.1038/nature03132

Leinekugel, X., Khazipov, R., Cannon, R., Hirase, H., Ben-Ari, Y., and Buzsáki, G. (2002). Correlated bursts of activity in the neonatal hippocampus in vivo. Science 296, 2049-2052. doi: 10.1126/science.1071111

Minlebaev, M., Colonnese, M., Tsintsadze, T., Sirota, A., and Khazipov, R. (2011). Early gamma oscillations synchronize developing thalamus and cortex. Science 334, 226-229. doi: 10.1126/science.1210574

Paxinos, G., Tork, I., Tecott, L. H., and Valentino, K. L. (1991). Atlas of the Developing Rat Brain. San Diego, CA: Academic Press.

Paxinos, G., and Watson, C. (2007). The Rat Brain in Stereotaxic Coordinates, 6th $E d n$. San Diego, CA: Academic Press.

Petit, L. F., Jalabert, M., Buhler, E., Malvache, A., Peret, A., Chauvin, Y., et al. (2014). Normotopic cortex is the major contributor to epilepsy in experimental double cortex. Ann. Neurol. 76, 428-442. doi: 10.1002/ana.24237 
Ramachandra, R., and Subramanian, T. (2011). Atlas of the Neonatal Rat Brain. Boca Raton, FL: CRC Press.

Sherwood, N., and Timiras, P. (1970). A Stereotaxic Atlas of the Developing Rat Brain. Berkeley: University of California Press.

Tiriac, A., Uitermarkt, B. D., Fanning, A. S., Sokoloff, G., and Blumberg, M. S. (2012). Rapid whisker movements in sleeping newborn rats. Curr. Biol. 22, 2075-2080. doi: 10.1016/j.cub.2012. 09.009

Tyzio, R., Nardou, R., Ferrari, D. C., Tsintsadze, T., Shahrokhi, A., Eftekhari, S., et al. (2014). Oxytocin-mediated GABA inhibition during delivery attenuates autism pathogenesis in rodent offspring. Science 343, 675-679. doi: $10.1126 /$ science. 1247190

Workman, A. D., Charvet, C. J., Clancy, B., Darlington, R. B., and Finlay, B. L. (2013). Modeling Transformations of neurodevelopmental sequences across Mammalian species. J. Neurosci. 33, 7368-7383. doi: 10.1523/JNEUROSCI.5746-12.2013
Yang, J. W., An, S., Sun, J. J., Reyes-Puerta, V., Kindler, J., Berger, T., et al. (2013). Thalamic network oscillations synchronize ontogenetic columns in the newborn rat barrel cortex. Cereb. Cortex 23, 1299-1316. doi: $10.1093 /$ cercor/bhs 103

Conflict of Interest Statement: The authors declare that the research was conducted in the absence of any commercial or financial relationships that could be construed as a potential conflict of interest.

Copyright (c) 2015 Khazipov, Zaynutdinova, Ogievetsky, Valeeva, Mitrukhina, Manent and Represa. This is an open-access article distributed under the terms of the Creative Commons Attribution License (CC BY). The use, distribution or reproduction in other forums is permitted, provided the original author(s) or licensor are credited and that the original publication in this journal is cited, in accordance with accepted academic practice. No use, distribution or reproduction is permitted which does not comply with these terms. 